\title{
A CLASS OF REGULAR FUNCTIONS CONTAINING SPIRALLIKE AND CLOSE-TO-CONVEX FUNCTIONS
}

\author{
M. R. ZIEGLER( ${ }^{\text {B) }}$
}

\begin{abstract}
A class of functions $\mathscr{A}$ is defined which contains the spirallike and close-to-convex functions. By decomposing $\mathscr{A}$ into subclasses in a natural way, some basic properties of $\mathscr{A}$ and these subclasses are determined, including solutions to extremal problems; distortion theorems; coefficient inequalities; and the radii of convexity and close-to-convexity.
\end{abstract}

1. Introduction. If $f(z)=z+a_{2} z^{2}+\cdots$ is regular in the open unit disk $\Delta=\{z:|z|<1\}$ and satisfies the condition

$$
\operatorname{Re}\left[e^{i \alpha} z f^{\prime}(z) / f(z)\right] \geqq 0, \quad z \text { in } \Delta,
$$

for some real $\alpha,|\alpha|<\pi / 2$, then $f(z)$ is univalent in $\Delta$ [20]. Such functions are called "spirallike" [8]. Let $\mathscr{F}_{\alpha}$ denote the class of all functions $f(z)$ satisfying the above conditions for a given $\alpha$ and let $\mathscr{F}=\bigcup \mathscr{F}_{\alpha}(|\alpha|<\pi / 2)$.

If $\alpha=0$, then (1.1) implies that $f(z)$ is a starlike function [11], i.e. $\mathscr{F}_{0}$ is the class $\mathscr{S}^{*}$ of regular univalent functions that map $\Delta$ onto a domain starlike with respect to the origin. It follows from (1.1) that $f(z)$ is in $\mathscr{F}_{\alpha}$ if and only if there is a function $s(z)$ in $\mathscr{S}^{*}$ such that

$$
f(z)=z[s(z) / z]^{\cos \alpha \exp (-i \alpha)}, \quad z \text { in } \Delta,
$$

where $[s(z) / z]^{\cos \alpha \exp (-i \alpha)}=1$ at $z=0$.

A function $f(z)=z+a_{2} z^{2}+\cdots$, regular in $\Delta$, is said to be close-to-convex, denoted $f(z) \in \mathscr{C}$, if there is an $s(z)$ in $\mathscr{S}^{*}$ and a complex number $\varepsilon,|\varepsilon|=1$, such that

$$
\operatorname{Re}\left[\varepsilon z f^{\prime}(z) / s(z)\right] \geqq 0, \quad z \text { in } \Delta .
$$

Kaplan has shown that functions in $\mathscr{C}$ are univalent and has given a geometric interpretation of (1.3) [6]. The purpose of this paper is to generalize the concept of close-to-convexity by formally replacing $s(z)$ in (1.3) with a spirallike function and to investigate the properties of the functions obtained in this manner.

DEFinition. $f(z)$ is regular in $\Delta, f(0)=0$ and $f^{\prime}(0)=1 . f(z)$ is in $\mathscr{A}$ if and only if there is a $g(z)$ in $\mathscr{F}$ and a complex number $\varepsilon,|\varepsilon|=1$, such that

$$
\operatorname{Re}\left[\varepsilon z f^{\prime}(z) / g(z)\right] \geqq 0, \quad z \text { in } \Delta .
$$

Received by the editors February 4, 1971 and, in revised form, June 29, 1971.

AMS 1969 subject classifications. Primary 3042.

Key words and phrases. Spirallike, close-to-convex.

(1) This work formed part of the author's Ph.D. dissertation which was written under the supervision of Professor R. J. Libera at the University of Delaware.

Copyright (c) 1972, American Mathematical Society 
In studying the properties of functions in $\mathscr{A}$, it is useful to consider the following subclasses: let $\mathscr{A}_{\alpha}$ represent the class of all functions $f(z)$ satisfying (1.4) for some $g(z)$ in $\mathscr{F}_{\alpha}$ and let $\mathscr{A}_{\alpha, \beta}$ be the class of functions satisfying (1.4) for $g(z) \in \mathscr{F}_{\alpha}$ and a fixed $\varepsilon, \varepsilon=e^{i \beta}$. (1.4) implies that $|\beta| \leqq \pi / 2$, thus $\mathscr{A}_{\alpha}=\bigcup \mathscr{A}_{\alpha, \beta}(|\beta| \leqq \pi / 2)$, and $\mathscr{A}=\bigcup \mathscr{A}_{\alpha}(|\alpha|<\pi / 2)$. A comparison of (1.4) with (1.3) shows $\mathscr{C}=\mathscr{A}_{0}$ and letting $g(z)=f(z)$ and $\beta=\alpha$ gives $\mathscr{F}_{\alpha} \subset \mathscr{A}_{\alpha, \alpha}$.

Let $\mathscr{G}_{\alpha}$ be the class of functions $f(z)$ with the property that $z f^{\prime}(z)$ is in $\mathscr{F}_{\alpha}$ [9], [18]. If we let $g(z)=z f^{\prime}(z)$, then (1.4) is satisfied for each $f(z)$ in $\mathscr{G}_{\alpha}$ and any $\varepsilon, \varepsilon=e^{i \beta}$, $|\beta| \leqq \pi / 2$, i.e. $\mathscr{G}_{\alpha} \subset \mathscr{A}_{\alpha, \beta}$ for all $\beta$. Robertson shows that $\mathscr{G}_{\alpha}$ contains functions which are not univalent whenever $\cos \alpha \geqq \frac{1}{2}$ [18], and hence so does $\mathscr{A}_{\alpha, \beta}$. Consequently, if $\mathscr{S}$ represents the class of regular univalent functions $f(z)$ normalized by $f(0)=0$ and $f^{\prime}(0)=1$, then $\mathscr{A} \notin \mathscr{S}$, however $\mathscr{A} \cap \mathscr{S}$ is a class of univalent functions containing $\mathscr{C} \cup \mathscr{F}$. We show in a later section that $\mathscr{S} \notin \mathscr{A}$, hence $\mathscr{A} \cap \mathscr{S}$ is a proper subset of $\mathscr{S}$.

If $\varepsilon=e^{i \beta}$ and $|\beta|=\pi / 2$, then (1.4) implies $\mathscr{A}_{\alpha, \beta}=\mathscr{G}_{\alpha}$. Since $\mathscr{G}_{\alpha} \subset \mathscr{A}_{\alpha, \beta}$ for all $\beta$, $|\beta| \leqq \pi / 2$, we will consider only $|\beta|<\pi / 2$.

2. Extremal problems. Let $\mathscr{P}$ represent the class of functions $p(z)$ regular in $\Delta$ and satisfying $\operatorname{Re}[p(z)] \geqq 0, z$ in $\Delta$, and $p(0)=1$. Thus we have $f(z)$ in $\mathscr{A}_{\alpha, \beta}$ if and only if there exists $g(z)$ in $\mathscr{F}_{\alpha}$ and $p(z)$ in $\mathscr{P}$ such that

$$
z f^{\prime}(z)=e^{-i \beta} g(z)[\cos \beta p(z)+i \sin \beta] .
$$

$\mathscr{F}_{\alpha}$ and $\mathscr{P}$ are both normal and compact families of analytic functions, hence $\mathscr{A}_{\alpha, \beta}$ is also normal and compact. Consequently, if $G\left(w_{1}, \ldots, w_{n+1}\right)$ is analytic in $C^{n+1}$ and $c$ is a fixed point in $\Delta$, then there exists $f_{0}(z)$ in $\mathscr{A}_{\alpha, \beta}$ such that the functional $\operatorname{Re}\left\{G\left[f^{\prime}(c), \ldots, f^{(n)}(c), c\right]\right\}$ assumes its maximal value over $\mathscr{A}_{\alpha, \beta}$ at $f_{0}(z)$. The following two theorems enable us to characterize $f_{0}(z)$. The first is due to Pinchuk [13] and the second to Sakaguchi [19].

THEOREM A. If $F\left(w_{0}, \ldots, w_{n+1}\right)$ is analytic and nonconstant in $C^{n+2}$ and $c \neq 0$ is in $\Delta$, then the functional $\operatorname{Re}\left\{F\left[f(c), \ldots, f^{(n)}(c), c\right]\right\}$ attains its maximum in $\mathscr{F}_{\alpha}$ only for a function of the form

$$
f(z)=z \prod_{j=1}^{N}\left(1-\varepsilon_{j} z\right)^{-2 \mu_{j} \cos \alpha \exp (-i \alpha)}
$$

where $\left|\varepsilon_{j}\right|=1$ and $\mu_{j} \geqq 0, j=1, \ldots, N ; \sum_{j=1}^{N} \mu_{j}=1$; and $N \leqq n+1$.

THEOREM B. If $F\left(w_{0}, \ldots, w_{n}\right)$ is analytic and nonconstant in $\operatorname{Re} w_{0} \geqq 0,\left|w_{k}\right|<+\infty$, $k=1, \ldots, n$, and $c \neq 0$ is in $\Delta$, then the functional $\operatorname{Re}\left\{F\left[p(c), p^{\prime}(c), \ldots, p^{(n)}(c)\right]\right\}$ attains its maximum in $\mathscr{P}$ only for a function of the form

$$
p(z)=\sum_{k=1}^{M} \eta_{k} \frac{1+\xi_{k} z}{1-\xi_{k} z}
$$

where $\left|\xi_{k}\right|=1$ and $\eta_{k} \geqq 0, k=1, \ldots, M ; \sum_{k=1}^{M} \eta_{k}=1 ;$ and $M \leqq n+1$. 
THEOREM 1. If $G\left(w_{1}, \ldots, w_{n+1}\right)$ is analytic and nonconstant in $C^{n+1}$ and $c \neq 0$ is in $\Delta$, then the functional $\operatorname{Re}\left\{G\left[f^{\prime}(c), \ldots, f^{(n)}(c), c\right]\right\}$ attains its maximum in $\mathscr{A}_{\alpha, \beta}$ only for a function of the form

$$
f^{\prime}(z)=\left[\prod_{j=1}^{N}\left(1-\varepsilon_{j} z\right)^{-2 \mu_{j} \cos \alpha \exp (-i \alpha)}\right] \sum_{k=1}^{M} \eta_{k} \frac{1+e^{-2 i} \beta \xi_{k} z}{1-\xi_{k} z}
$$

where $\left|\varepsilon_{j}\right|=1$ and $\mu_{j} \geqq 0, j=1, \ldots, N ;\left|\xi_{k}\right|=1$ and $\eta_{k} \geqq 0, k=1, \ldots, M ; \sum_{j=1}^{N} \mu_{j}=1$ and $\sum_{k=1}^{M} \eta_{k}=1 ; N \leqq n$ and $M \leqq n$.

Proof. By virtue of the compactness of $\mathscr{A}_{\alpha, \beta}$, the extremal problem

$$
\max _{f(z) \in \mathscr{A}_{\alpha, \beta}} \operatorname{Re}\left\{G\left[f^{\prime}(c), \ldots, f^{(n)}(c), c\right]\right\}
$$

has a solution in $\mathscr{A}_{\alpha, \beta}$. Let $f_{0}(z)$ be any solution to $(2.3)$ and let $g_{0}(z)$ in $\mathscr{F}_{\alpha}$ and $p_{0}(z)$ in $\mathscr{P}$ be chosen so that (2.1) is satisfied. For each $g(z)$ in $\mathscr{F}_{\alpha}$ define $H\left(w_{0}, \ldots, w_{n}\right)$ by

$$
H\left[g(c), \ldots, g^{(n-1)}(c), c\right]=G\left[\hat{f}^{\prime}(c), \ldots, \hat{f}^{(n)}(c), c\right]
$$

where $z \hat{f}^{\prime}(z)=e^{-i \beta} g(z)\left[\cos \beta p_{0}(z)+i \sin \beta\right]$. Using the extremal property of $f_{0}(z)$ we have

$$
\begin{aligned}
\operatorname{Re}\{H[g(c) & \left.\left.\ldots, g^{(n-1)}(c), c\right]\right\}=\operatorname{Re}\left\{G\left[\hat{f}^{\prime}(c), \ldots, \hat{f}^{(n)}(c), c\right]\right\} \\
& \leqq \operatorname{Re}\left\{G\left[f_{0}^{\prime}(c), \ldots, f_{0}^{(n)}(c), c\right]\right\}=\operatorname{Re}\left\{H\left[g_{0}(c), \ldots, g_{0}^{(n-1)}(c), c\right]\right\} .
\end{aligned}
$$

Thus $g_{0}(z)$ is a solution to an extremal problem over $\mathscr{F}_{\alpha}$ and must be of the form given in Theorem A. In a similar manner, by fixing $g_{0}(z)$ and allowing $p_{0}(z)$ to vary over the class $\mathscr{P}$, we are able to conclude that $p_{0}(z)$ is of the form given in Theorem B and hence $f_{0}(z)$ satisfies (2.2).

In the same manner, using well-known properties of $\mathscr{F}_{\alpha}$ and $\mathscr{P}$ (see [2], [4], [5], [16], [19]), we obtain the following theorem.

THEOREM 2. Let $G\left(w_{1}, \ldots, w_{n}\right)$ be analytic and nonconstant in $C^{n}$ and let $f(z)$ $=z+a_{2} z^{2}+\cdots+a_{n} z^{n}+\cdots$ belong to $\mathscr{A}_{\alpha, \beta}$. The functional $\operatorname{Re}\left[G\left(a_{2}, \ldots, a_{n+1}\right)\right]$ is maximized over the class $\mathscr{A}_{\alpha, \beta}$ only by functions of the form (2.2).

3. Distortion theorems and coefficient inequalities. Theorem 1 indicates that to determine sharp bounds for $\arg f^{\prime}(z)$ and $\left|f^{\prime}(z)\right|$ when $f(z)$ is in $\mathscr{A}_{\alpha, \beta}$, it is sufficient to obtain these bounds for the function defined by (2.2). As the expressions involved are rather complicated and the techniques used are elementary, we will consider here only the lower bound for $\arg f^{\prime}(z)$ which will be used in some subsequent work.

THEOREM 3. If $f(z)$ belongs to $\mathscr{A}_{\alpha, \beta}$ and $|z|=r$, then

$$
\begin{aligned}
\arg \left[f^{\prime}(z)\right] \geqq & -2 \cos ^{2} \alpha \arcsin (r \cos \alpha)+\sin 2 \alpha \ln \left(\left(1-r^{2} \cos ^{2} \alpha\right)^{1 / 2}-r \sin \alpha\right) \\
& -2 \arcsin \left[r \cos \beta\left(1-2 r \sin \beta+r^{2}\right)^{-1 / 2}\right]
\end{aligned}
$$

with equality occurring only for functions of the form (2.2) with $N=M=1$. 
Proof. If $f(z)$ is defined by (2.2) and $N=M=1$, then

$$
\arg \left[f^{\prime}(z)\right]=-2 \cos \alpha \arg \left[\left(1-r e^{i \theta}\right)^{\exp (-i \alpha)}\right]+\arg \left[\left(1+r e^{i(\phi-2 \beta)}\right) /\left(1-r e^{i \phi}\right)\right],
$$

where $\varepsilon_{1} z=r e^{i \theta}$ and $\xi_{1} z=r e^{i \phi}$. Let $V(\theta)$ be defined by

$$
\begin{aligned}
V(\theta)= & \arg \left[\left(1-r e^{i \theta}\right)^{\exp (-i \alpha)}\right] \\
= & \cos \alpha \operatorname{arc} \sin \left[-r \sin \theta\left(1-2 r \cos \theta+r^{2}\right)^{-1 / 2}\right] \\
& -\sin \alpha \ln \left[\left(1-2 r \cos \theta+r^{2}\right)^{1 / 2}\right] .
\end{aligned}
$$

$V^{\prime}(\theta)=r[r \cos \alpha-\cos (\theta-\alpha)] /\left(1-2 r \cos \theta+r^{2}\right)$ hence $V(\theta)$ assumes its maximum value at $\theta=\theta_{0}$ where $\theta_{0}$ satisfies

$$
\cos \left(\theta_{0}-\alpha\right)=r \cos \alpha ; \quad \sin \left(\theta_{0}-\alpha\right)=-\left(1-r^{2} \cos ^{2} \alpha\right)^{1 / 2} .
$$

Expanding the equations in (3.3) and solving for $\cos \theta_{0}$ and $\sin \theta_{0}$ yields

$$
\begin{aligned}
\cos \theta_{0} & =r \cos ^{2} \alpha+\sin \alpha\left(1-r^{2} \cos ^{2} \alpha\right)^{1 / 2}, \\
\sin \theta_{0} & =r \sin \alpha \cos \alpha-\cos \alpha\left(1-r^{2} \cos ^{2} \alpha\right)^{1 / 2}
\end{aligned}
$$

and using (3.2) and (3.4) to evaluate $V\left(\theta_{0}\right)$ gives

$$
V(\theta) \leqq V\left(\theta_{0}\right)=\cos \alpha \arcsin (r \cos \alpha)-\sin \alpha \ln \left(\left(1-r^{2} \cos ^{2} \alpha\right)^{1 / 2}-r \sin \alpha\right) .
$$

In a similar manner, if

$$
\begin{aligned}
u(\phi) & =\arg \left[\left(1+r e^{i(\phi-2 \beta)}\right) /\left(1-r e^{i \phi}\right)\right] \\
& =\arcsin \left[\frac{r \sin (\phi-2 \beta)}{\left(1-2 r \cos (\phi-2 \beta)+r^{2}\right)^{1 / 2}}\right]+\arcsin \left[\frac{r \sin \phi}{\left(1-2 r \cos \phi+r^{2}\right)}\right],
\end{aligned}
$$

then we find

$$
u(\phi) \geqq u[\beta+(3 \pi / 2)]=-2 \arcsin \left[r \cos \beta\left(1+2 r \sin \beta+r^{2}\right)^{-1 / 2}\right] .
$$

Thus (3.5) and (3.6) together imply that $\arg \left[f^{\prime}(z)\right]$ satisfies the bound in (3.1). Furthermore, if $g(z)$ is an $\mathscr{A}_{\alpha, \beta}$, then by Theorem 1 for each $z$, with $|z|=r, \arg \left[g^{\prime}(z)\right]$ $\geqq \arg \left[f^{\prime}(z)\right]$ and hence $\min \arg \left[g^{\prime}(z)\right](|z|=r) \geqq \min \arg \left[f^{\prime}(z)\right](|z|=r)$. Thus the bound given in (3.1) is valid for all functions in $\mathscr{A}_{\alpha, \beta}$ and equality occurs only for $f(z)$ defined by (2.2) with an appropriate choice of $\varepsilon_{1}$ and $\xi_{1}$.

CoRollaRY 1. If $f(z)$ is in $\mathscr{A}_{\alpha}$ and $|z|=r$, then

$$
\begin{aligned}
\arg \left[f^{\prime}(z)\right] \geqq & -2 \cos ^{2} \alpha \arcsin (r \cos \alpha) \\
& +\sin 2 \alpha \ln \left(\left(1-r^{2} \cos ^{2} \alpha\right)^{1 / 2}-r \sin \alpha\right)-2 \arcsin r .
\end{aligned}
$$

This result is sharp.

Proof. $f(z)$ in $\mathscr{A}_{\alpha}$ implies $f(z)$ belongs to $\mathscr{A}_{\alpha, \beta}$ for some $\beta$, hence (3.1) together with the fact that

$$
\arcsin \left[r \cos \beta\left(1+2 r \sin \beta+r^{2}\right)^{-1 / 2}\right] \leqq \arcsin r
$$

for all $\beta,|\beta|<\pi / 2$, yields (3.7). Equality occurs in (3.7) for $f(z)$ defined by (2.2) with $\sin \beta=-r$. 
THEOREM 4. If $f(z)$ is in $\mathscr{A}$ and $|z|=r$, then

$$
(1-r) /(1+r)^{3} \leqq\left|f^{\prime}(z)\right| \leqq(1+r) /(1-r)^{3}
$$

and

$$
|f(z)| \leqq r /(1-r)^{2} .
$$

Both (3.8) and (3.9) are sharp.

Proof. Since $f(z)$ is in $\mathscr{A}$, there is a $g(z)$ in $\mathscr{F}, p(z)$ in $\mathscr{P}$ and a real number $\beta$, $|\beta|<\pi / 2$, such that $(2.1)$ is satisfied. $q(z)=e^{-i \beta}[\cos \beta p(z)+i \sin \beta]$ is subordinate to $q_{0}(z)=\left(1+e^{-2 i \beta} z\right) /(1-z)$, hence

$$
\frac{1-r}{1+r} \leqq \frac{\left|1+r^{2} e^{-2 i \beta}\right|-2 r \cos \beta}{1-r^{2}} \leqq|q(z)| \leqq \frac{\left|1+r^{2} e^{-2 t \beta}\right|+2 r \cos \beta}{1-r^{2}} \leqq \frac{1+r}{1-r}
$$

Since $g(z)$ is univalent, the well-known bounds on $|g(z)|$ [11], together with (3.10), imply (3.8). (3.9) then follows from (3.8) by an integration. Equality is obtained in (3.8) and (3.9) only for $f(z)=z /(1-\varepsilon z)^{2},|\varepsilon|=1$.

It is interesting to note that the bounds given in (3.8) and (3.9) are also the sharp bounds for the class $\mathscr{S}$. To further investigate the relationship between $\mathscr{S}$ and $\mathscr{A}$ with regard to the growth of $\left|f^{\prime}(z)\right|$ and $|f(z)|$, it is useful to study the Hardy classes of $\mathscr{A}$. A function $f(z)$ regular in $\Delta$ belongs to the Hardy class $H^{\lambda}, \lambda>0$, if $\lim _{r \rightarrow 1^{-}} \int_{-\pi}^{\pi}\left|f\left(r e^{i \theta}\right)\right|^{\lambda} d \theta$ exists and is finite.

THEOREM 5. Let $f(z)$ belong to $\mathscr{A}_{\alpha}$ and choose $g(z)$ in $\mathscr{F}_{\alpha}$ and $p(z)$ in $\mathscr{P}$ so that (2.1) is satisfied. If $g(z) \neq z /(1-\mu z)^{2 \cos \alpha \exp (-i \alpha)},|\mu|=1$, then

(i) there exists $\varepsilon=\varepsilon(f)$ such that $f^{\prime}(z)$ is in $H^{\lambda}, \lambda=\left(2 \cos ^{2} \alpha+1\right)^{-1}+\varepsilon$;

(ii) there exists $\delta=\delta(f)$ such that $f(z)$ is in $H^{\lambda}, \lambda=\frac{1}{2} \sec ^{2} \alpha+\delta$.

If $g(z)=z /(1-\mu z)^{2 \cos \alpha \exp (-i \alpha)}$, then

(iii) $f^{\prime}(z)$ is in $H^{\lambda}$ whenever $\lambda<\left(2 \cos ^{2} \alpha+1\right)^{-1}$;

(iv) $f(z)$ is in $H^{\lambda}$ whenever $\lambda<\frac{1}{2} \sec ^{2} \alpha$.

Başgöze and Keogh have recently proven a similar result for $\mathscr{F}_{\alpha}[1]$, and Eenigenburg and Keogh have obtained Theorem 5 in the case $\alpha=0$ [3]. As the proof for general $\alpha$ is essentially the same as that given in [3], we will omit the proof of Theorem 5.

An immediate consequence of the above is that if $f(z)$ is in $\mathscr{A}$, then $f^{\prime}(z)$ is in $H^{\lambda}$ for all $\lambda$ satisfying $0<\lambda<\frac{1}{3}$. However, there exists a univalent function $f(z)$ such that $f^{\prime}(z)$ is not in $H^{\lambda}$ for any $\lambda>0$ [10], hence $\mathscr{S}$ is not a subclass of $\mathscr{A}$.

If $f(z)=z+a_{2} z^{2}+\cdots$ is close-to-convex in $\Delta$, Reade has shown that $\left|a_{n}\right| \leqq n$, $n=2,3, \ldots$ [15], and Pommerenke has proven that ||$a_{n}|-| a_{n+1}||<\left(3 e^{2}\right) / 4$ [14]. An examination of the proofs of these results allows us to conclude that these inequalities are also valid for the class $\mathscr{A}$, hence we have the following theorem. 
THEOREM 6. If $f(z)=z+a_{2} z^{2}+\cdots$ is in $\mathscr{A}$, then

$$
\left|a_{n}\right| \leqq n, \quad n=2,3, \ldots,
$$

and

$$
|| a_{n}|-| a_{n+1}|| \leqq\left(3 e^{2}\right) / 4, \quad n=2,3, \ldots
$$

Since $f(z)=z /(1-z)^{2}$ is in $\mathscr{A},(3.11)$ is sharp. The sharp bound for ||$a_{n}|-| a_{n+1}||$ is not known, even for the class $\mathscr{C}$. If $f(z)$ is restricted to $\mathscr{A}_{\alpha}$ or $\mathscr{A}_{\alpha, \beta}$, then (3.11) can be improved by making use of the coefficient bounds for $\mathscr{F}_{\alpha}$ [8], however we have not been able to determine the sharp bounds in either case.

4. Radii of convexity and close-to-convexity. If $f(z)$ is in $\mathscr{S}$ and $f_{*}(z)$ is defined by

$$
f_{*}(z)=\{f[(z+a) /(1+\bar{a} z)]-f(a)\} \mid f^{\prime}(a)\left(1-|a|^{2}\right), \quad a \text { and } z \text { in } \Delta,
$$

then $f_{*}(z)$ is in $\mathscr{S}$. (4.1) can be used to determine many properties of univalent functions. In this section we obtain an analogous transformation for functions in $\mathscr{A}_{\alpha}$ and use it to find the radius of convexity and the radius of close-to-convexity of $\mathscr{A}_{\alpha}$.

THEOREM 7. If $f(z)$ is in $\mathscr{A}_{\alpha}$ and $f_{*}(z)$ is defined by

$$
f_{*}^{\prime}(z)=f^{\prime}[(z+a) /(1+\bar{a} z)] / f^{\prime}(a)(1+\bar{a} z)^{\exp (-2 i \alpha)+1}, \quad z \text { and } a \text { in } \Delta,
$$

and $f(0)=0$, then $f_{*}(z)$ belongs to $\mathscr{A}_{\alpha}$.

Proof. There is a $g(z)$ in $\mathscr{F}_{\alpha}$ and a complex number $\varepsilon,|\varepsilon|=1$, such that $\operatorname{Re}\left[\varepsilon z f^{\prime}(z) / g(z)\right] \geqq 0$. If $g_{*}(z)$ is defined by

$$
g_{*}(z)=a z g[(z+a) /(1+\bar{a} z)] / g(a)(z+a)(1+\bar{a} z)^{\exp (-2 i \alpha)},
$$

then $g_{*}(z)$ is also in $\mathscr{F}_{\alpha}$ [9], [21]. Let $\varepsilon_{1}=e^{i \phi}$ where $\phi=\arg \left[\varepsilon a f^{\prime}(a) / g(a)\right],|\phi|<\pi / 2$. Then, with $w=(z+a) /(1+\bar{a} z)$,

$$
\operatorname{Re}\left[\varepsilon_{1} \frac{z f_{*}^{\prime}(z)}{g_{*}(z)}\right]=\operatorname{Re}\left[\varepsilon_{1} \frac{g(a)}{a f^{\prime}(a)} \frac{w f^{\prime}(w)}{g(w)}\right]=\left|\frac{g(a)}{a f^{\prime}(a)}\right| \operatorname{Re}\left[\varepsilon \frac{w f^{\prime}(w)}{g(w)}\right] \geqq 0
$$

and consequently $f_{*}(z)$ belongs to $\mathscr{A}_{\alpha}$.

Notice that $\varepsilon_{1}$ depends on the choice of $a$, hence it is not possible to conclude that $f_{*}(z) \in \mathscr{A}_{\alpha, \beta}$ whenever $f(z) \in \mathscr{A}_{\alpha, \beta}$. In order to apply (4.2) we will need the following lemma.

LEMMA 1. If $f(z)=z+a_{2} z^{2}+\cdots$ is in $\mathscr{A}_{\alpha}$, then $\left|a_{2}\right| \leqq \cos \alpha+1$. This inequality is sharp. 
Proof. Let $z f^{\prime}(z)=g(z) e^{-i \beta}[\cos \beta p(z)+i \sin \beta], g(z)$ in $\mathscr{F}_{\alpha}$ and $p(z)$ in $\mathscr{P}$. If $g(z)$ $=z+b_{2} z^{2}+\cdots$ and $p(z)=1+p_{1} z+\cdots$, then $\left|b_{2}\right| \leqq 2 \cos \alpha$ [8], and $\left|p_{1}\right| \leqq 2[11]$. Equating coefficients we have

$$
\left|a_{2}\right|=\frac{1}{2}\left|b_{2}+e^{-i \beta} \cos \beta p_{1}\right| \leqq \cos \alpha+\cos \beta
$$

from which the lemma follows. Equality is obtained when $g(z)=z /(1-z)^{2 \cos \alpha \exp (-\imath \alpha)}$, $p(z)=\left(1+e^{-i \alpha} z\right) /\left(1-e^{-i \alpha} z\right)$, and $\beta=0$.

THEOREM 8. The radius of convexity of $\mathscr{A}_{\alpha}$ is the smallest positive root $r_{0}$ of the equation

$$
1-2(1+\cos \alpha) r+\cos 2 \alpha r^{2}=0,
$$

and the radius of convexity of $\mathscr{A}$ is $2-3^{1 / 2}$.

Proof. Let $f(z)$ be in $\mathscr{A}_{\alpha}$ and let $f_{*}(z)=z+a_{2}^{*} z^{2}+\cdots$ be defined by (4.2). A calculation shows

$$
a_{2}^{*}=\frac{1}{2}\left[\left(1-|a|^{2}\right) f^{\prime \prime}(a) / f^{\prime}(a)-\bar{a}\left(e^{-2 i \alpha}+1\right)\right]
$$

and hence, if $|a|=r$, from Lemma 1 we have

$$
\left|\frac{a f^{\prime \prime}(a)}{f^{\prime}(a)}-\frac{\left(1+e^{-2 i \alpha}\right) r^{2}}{1-r^{2}}\right| \leqq \frac{2 r(1+\cos \alpha)}{1-r^{2}} .
$$

Letting $a=z$, it follows that for any $z$ with $|z|=r$

$$
\operatorname{Re}\left[1+z f^{\prime \prime}(z) / f^{\prime}(z)\right] \geqq\left[1-2 r(1+\cos \alpha)+r^{2} \cos 2 \alpha\right] /\left(1-r^{2}\right),
$$

consequently $f(z)$ maps $|z|<r$ onto a convex domain whenever $1-2 r(1+\cos \alpha)$ $+r^{2} \cos 2 \alpha \geqq 0$. The function $f(z)$ defined by (2.2) shows that this result is sharp. Furthermore, since $1-2 r(1+\cos \alpha)+r^{2} \cos 2 \alpha \geqq 1-4 r+r^{2}$ for all $\alpha,|\alpha|<\pi / 2$, and all $r, 0 \leqq r<1$, it follows that the sharp radius of convexity of $\mathscr{A}$ is the smallest positive root of $1-4 r+r^{2}=0$, namely $2-3^{1 / 2}$. It is interesting to note that $2-3^{1 / 2}$ is also the radius of convexity of $\mathscr{S}$.

Let $f(z)$ be regular in $\Delta$ and have a nonvanishing derivative there. Kaplan [6] has shown that a necessary and sufficient condition for $f(z)$ to map $|z|=r$ onto a close-to-convex curve is that

$$
\arg \left[z_{2} f^{\prime}\left(z_{2}\right)\right]-\arg \left[z_{1} f^{\prime}\left(z_{1}\right)\right] \geqq-\pi
$$

for all $z_{1}$ and $z_{2}$ with $\left|z_{1}\right|=r$ and $z_{2}=z_{1} e^{i \theta}, 0<\theta<2 \pi$. The radius of close-toconvexity of $\mathscr{A}_{\alpha}$ will then be the largest value of $r$ for which all functions $f(z)$ in $\mathscr{A}_{\alpha}$ satisfy (4.3). We determine this value as the solution of an equation depending on $r$ and $\alpha$. Some of the techniques used are similar to those employed by Krzyż to obtain the radius of close-to-convexity of $\mathscr{S}$ [7].

THEOREM 9. Let $\alpha \neq 0$, let $r_{0}$ be the radius of convexity of $\mathscr{A}_{\alpha}$, and let $r \in\left(r_{0}, 1\right)$. If $x_{0}$ is the positive root of

$$
p(x)=\left(1+r^{2}\right)\left(1-r^{2} \cos 2 \alpha\right)-2 r\left(1-r^{2}\right) x-4 r^{2} \sin ^{2} \alpha x^{2} ;
$$


$x_{1}=\min \left\{1, x_{0}\right\} ; x_{2}$ is the unique root in $\left(0, x_{1}\right)$ of

$$
\begin{aligned}
p_{2}(x)= & \left(1+r^{2}\right)\left(1-r^{2} \cos 2 \alpha\right)^{2}-4 r\left(1-r^{4}\right)\left(1-r^{2} \cos 2 \alpha\right) x \\
& -4 r^{2} \sin ^{2} \alpha\left(1+6 r^{2}-3 r^{4}+4 r^{4} \sin ^{2} \alpha\right) x^{2} \\
& +16 r^{3} \sin ^{2} \alpha\left(1-r^{2}\right) x^{3}+16 r^{4} \sin ^{2} \alpha x^{4} ;
\end{aligned}
$$

$\theta_{0}=2 \arccos x_{2}, 0<\theta_{0}<\pi ;$ and

$$
\begin{aligned}
\Delta(r)=\theta_{0} & +2 \cos ^{2} \alpha \arctan \left[r^{2} \sin \theta_{0} /\left(1-r^{2} \cos \theta_{0}\right)\right] \\
& -2 \cos ^{2} \alpha \arcsin \left\{r \cos \alpha\left[2\left(1-\cos \theta_{0}\right) /\left(1-2 r^{2} \cos \theta_{0}+r^{4}\right)\right]^{1 / 2}\right\} \\
& +\sin 2 \alpha \ln \left\{\left[1-2 r^{2}\left(\sin ^{2} \alpha \cos \theta_{0}+\cos ^{2} \alpha\right)+r^{4}\right]^{1 / 2}\right. \\
& \left.-r \sin \alpha\left[2\left(1-\cos \theta_{0}\right)\right]^{1 / 2}\right\} \\
& -2 \arcsin \left\{r\left[2\left(1-\cos \theta_{0}\right) /\left(1-2 r^{2} \cos \theta_{0}+r^{4}\right)\right]^{1 / 2}\right\} \\
& -\sin 2 \alpha \ln \left(1-r^{2}\right) ;
\end{aligned}
$$

then the radius of close-to-convexity of $\mathscr{A}_{\alpha}$ is the unique root of the equation $\Delta(r)=-\pi$.

Proof. Let $\Delta(r, \theta)=\inf \arg \left[z_{2} f^{\prime}\left(z_{2}\right) / z_{1} f^{\prime}\left(z_{1}\right)\right]\left(f(z) \in \mathscr{A}_{\alpha}\right)$ where the argument is chosen so as to vary continuously from an initial value of zero. If

$$
\zeta=\left(z-z_{1}\right) /\left(1-\bar{z}_{1} z\right) \text { and } \zeta_{0}=\left(z_{2}-z_{1}\right) /\left(1-\bar{z}_{1} z_{2}\right)
$$

and if $f_{*}(\zeta)$ is defined by

$$
f_{*}^{\prime}(\zeta)=f^{\prime}\left[\left(\zeta+z_{1}\right) /\left(1+\bar{z}_{1} \zeta\right)\right] / f^{\prime}\left(z_{1}\right)\left(1+\bar{z}_{1} \zeta\right)^{\exp (-2 i \alpha)+1}, \quad f_{*}(0)=0,
$$

then $f_{*}(\zeta)$ is in $\mathscr{A}_{\alpha}$ and

$$
f_{*}^{\prime}\left(\zeta_{0}\right)=\left[f^{\prime}\left(z_{2}\right) / f^{\prime}\left(z_{1}\right)\right]\left[\left(1-\bar{z}_{1} z_{2}\right) /\left(1-\left|z_{1}\right|^{2}\right)\right]^{\exp (-2 i \alpha)+1} .
$$

Thus we have

$$
\Delta(r, \theta)=\arg \left\{\left(z_{2} / z_{1}\right)\left[\left(1-\left|z_{1}\right|_{\cdot}^{2}\right) /\left(1-\bar{z}_{1} z_{2}\right)\right]^{\exp (-2 i \alpha)+1}\right\}+\inf _{f_{\bullet}(\zeta) \in \mathscr{A}_{\alpha}} \arg \left[f_{*}^{\prime}\left(\zeta_{0}\right)\right] .
$$

Using the sharp lower bound for $\arg \left[f_{*}^{\prime}\left(\zeta_{0}\right)\right]$ given in Corollary 1 , we can express $\Delta(r, \theta)$ explicitly as a function of $r$ and $\theta$. A brief calculation shows

$$
\begin{aligned}
& \arg \left\{\left[\left(1-\left|z_{1}\right|^{2}\right) /\left(1-\bar{z}_{1} z_{2}\right)\right]^{\exp (-2 i \alpha)+1}\right\} \\
&= 2 \cos ^{2} \alpha \arctan \left[r^{2} \sin \theta /\left(1-r^{2} \cos \theta\right)\right] \\
&-\sin 2 \alpha \ln \left[\left(1-r^{2}\right) /\left(1-2 r^{2} \cos \theta+r^{4}\right)^{1 / 2}\right]
\end{aligned}
$$

and

$$
\left|\zeta_{0}\right|=r\left[2(1-\cos \theta) /\left(1-2 r^{2} \cos \theta+r^{4}\right)\right]^{1 / 2} \text {. }
$$

Thus, using (3.7), (4.6) and (4.7) in (4.5) we can write

$$
\begin{aligned}
\Delta(r, \theta)=\theta & +2 \cos ^{2} \alpha \arctan \left[r^{2} \sin \theta /\left(1-r^{2} \cos \theta\right)\right] \\
& -2 \cos ^{2} \alpha \arcsin \left\{r \cos \alpha\left[2(1-\cos \theta) /\left(1-2 r^{2} \cos \theta+r^{4}\right)\right]^{1 / 2}\right\} \\
& +\sin 2 \alpha \ln \left\{\left[1-2 r^{2}\left(\sin ^{2} \alpha \cos \theta+\cos ^{2} \alpha\right)+r^{4}\right]^{1 / 2}\right. \\
& \left.-r \sin \alpha[2(1-\cos \theta)]^{1 / 2}\right\} \\
& -2 \arcsin \left\{r\left[2(1-\cos \theta) /\left(1-2 r^{2} \cos \theta+r^{4}\right)\right]^{1 / 2}\right\} \\
& -\sin 2 \alpha \ln \left(1-r^{2}\right) .
\end{aligned}
$$


Furthermore, for a fixed $z_{1}$ and $z_{2}$, if $f_{*}(z)$ is the function which gives equality in (3.7) and $f(z)$ is defined by

$$
f^{\prime}(z)=f_{*}^{\prime}\left[\left(z-z_{1}\right) /\left(1-\bar{z}_{1} z\right)\right] / f_{*}^{\prime}\left(-z_{1}\right)\left(1-\bar{z}_{1} z\right)^{\exp (-2 i \alpha)+1}, \quad f(0)=0,
$$

then $\arg \left\{z_{2} f^{\prime}\left(z_{2}\right) / z_{1} f^{\prime}\left(z_{1}\right)\right\}=\Delta(r, \theta)$; that is, for this function $f(z)$, equality occurs in (4.8).

If $\Delta(r)=\inf \Delta(r, \theta)(0<\theta<2 \pi)$, then $\Delta(r)$ is a decreasing function of $r$, and the radius of close-to-convexity of $\mathscr{A}_{\alpha}$ is the solution of the equation $\Delta(r)=-\pi$. The remainder of this argument is concerned with determining $\Delta(r)$ and showing that the equation $\Delta(r)=-\pi$ does have a solution. In what follows we shall restrict $r$ to the interval $\left(r_{0}, 1\right)$, where $r_{0}$ is the radius of convexity of $\mathscr{A}_{\alpha}$, since $\Delta(r) \geqq 0$ for $r \leqq r_{0}$.

Differentiating (4.8) with respect to $\theta$, we find

$$
\begin{aligned}
\partial \Delta(r, \theta) / \partial \theta= & 1+2 \cos ^{2} \alpha r^{2}\left(\cos \theta-r^{2}\right) /\left(1-2 r^{2} \cos \theta+r^{4}\right) \\
& -2 r \cos \alpha \sin \theta \frac{\left[1-2 r^{2}\left(\sin ^{2} \alpha \cos \theta+\cos ^{2} \alpha\right)+r^{4}\right]^{1 / 2}}{[2(1-\cos \theta)]^{1 / 2}\left(1-2 r^{2} \cos \theta+r^{4}\right)} \\
& -2 r \sin \theta\left(1-r^{2}\right) /[2(1-\cos \theta)]^{1 / 2}\left(1-2 r^{2} \cos \theta+r^{4}\right) .
\end{aligned}
$$

Putting $x=\cos (\theta / 2), 0<\theta<2 \pi$, we have $\sin \theta /[2(1-\cos \theta)]^{1 / 2}=x$ and $\cos \theta=2 x^{2}$ -1 , hence (4.9) can be written as $\partial \Delta(r, \theta) / \partial \theta=[p(x)-2 r x \cos \alpha k(x)] / g(x)$ where

$$
\begin{aligned}
& p(x)=\left(1+r^{2}\right)\left(1-r^{2} \cos 2 \alpha\right)-2 r\left(1-r^{2}\right) x-4 r^{2} \sin ^{2} \alpha x^{2}, \\
& k(x)=\left[1-2 r^{2} \cos 2 \alpha+r^{4}-4 r^{2} \sin ^{2} \alpha x^{2}\right]^{1 / 2}, \text { and } \\
& g(x)=\left(1+r^{2}\right)^{2}-4 r^{2} x^{2} .
\end{aligned}
$$

Since $g(x)>0$ for $0 \leqq r<1$ and $|x| \leqq 1$, the zeros of $\partial \Delta(r, \theta) / \partial \theta$ will be determined by the zeros of $p_{1}(x)=p(x)-2 r x \cos \alpha k(x)$. A simple calculation shows $p(x)>0$ for $x \in[-1,0]$ and clearly $-2 r x \cos \alpha k(x)>0$ for $x \in[-1,0]$, hence $p_{1}(x)$ has no zeros in $[-1,0]$. If $x_{0}$ is the positive zero of $p(x)$ and $x_{1}=\min \left\{1, x_{0}\right\}$, then any zeros of $p_{1}(x)$ must lie in the interval $\left(0, x_{1}\right)$, and furthermore, for $0<x<x_{1}$, the zeros of $p_{1}(x)$ will be identical with the zeros of $p_{2}(x)$ where

$$
\begin{aligned}
p_{2}(x)= & p(x)^{2}-4 r^{2} x^{2} \cos ^{2} \alpha k(x)^{2} \\
= & \left(1+r^{2}\right)^{2}\left(1-r^{2} \cos 2 \alpha\right)^{2}-4 r\left(1-r^{4}\right)\left(1-r^{2} \cos 2 \alpha\right) x \\
& -\left[4 r^{2} \sin ^{2} \alpha\left(1+6 r^{2}-3 r^{4}\right)+16 r^{6} \sin ^{4} \alpha\right] x^{2} \\
& +16 r^{3} \sin ^{2} \alpha\left(1-r^{2}\right) x^{3}+16 r^{4} \sin ^{2} \alpha x^{4} .
\end{aligned}
$$

The coefficients of $p_{2}(x)$ change sign twice, hence $p_{2}(x)$ has either zero or two positive roots.

Suppose now that $x_{0}$, the positive root of $p(x)$, lies in $(0,1]$, i.e. $x_{0}=x_{1}$. A brief calculation shows this will occur only when $r \geqq r_{1}$ where $r_{1}$ is the smallest positive root of the equation $1-2 r+r^{2} \cos 2 \alpha=0$. Hence, for $r \in\left[r_{1}, 1\right), p_{2}\left(x_{0}\right)$ $=-4 r^{2} x_{0}^{2} \cos ^{2} \alpha k\left(x_{0}\right)^{2}<0$ and, since $p_{2}(0)>0$, it follows that $p_{2}(x)$ has at least one root in $0<x<x_{0}=x_{1}$. But $p_{2}(x)$ has at most two roots for all $x>0$, thus there is exactly one simple root in $\left(0, x_{1}\right)$. 
If $x_{0}>1$, then $x_{1}=1$ and $r<r_{1}$. The radius of convexity $r_{0}$ of $\mathscr{A}_{\alpha}$ was shown to be the smallest positive root of the equation $1-2 r(1+\cos \alpha)+r^{2} \cos \alpha=0$. Clearly $r_{0}<r_{1}$ and for $r \in\left(r_{0}, r_{1}\right)$ we have $1-2 r+r^{2} \cos 2 \alpha>0$ and $1-2 r(1+\cos \alpha)$ $+r^{2} \cos 2 \alpha<0$. A calculation shows

$$
p_{2}(1)=\left(1-r^{2}\right)^{2}\left[1-2 r(1+\cos \alpha)+\cos 2 \alpha r^{2}\right]\left[1-2 r+\cos 2 \alpha r^{2}+2 r \cos \alpha\right]<0
$$

for $r_{0}<r<r_{1}$ and, as before, $p_{2}(x)$ must have a simple root in $\left(0, x_{1}\right)$.

It follows then that $\partial \Delta(r, \theta) / \partial \theta$ has one simple zero for all $r$ in $\left(r_{0}, 1\right)$ and thus $\Delta(r, \theta)$ assumes its minimum at $\theta=\theta_{0}$ where $\theta_{0}=2 \operatorname{arc} \cos x_{2}$ and $x_{2}$ is the unique root of $p_{2}(x)$ in $\left(0, x_{1}\right)$. Hence $\Delta(r)=\Delta\left(r, \theta_{0}\right)$ and letting $\theta=\theta_{0}$ in (4.8) yields the expression given for $\Delta(r)$ in (4.4). $\Delta(r)$ is a decreasing function of $r, \Delta\left(r_{0}\right)=0$, and an examination of (4.4) shows $\Delta(r) \rightarrow-\infty$ as $r \rightarrow 1^{-}$, so there is a unique solution $r_{\alpha}$ to the equation $\Delta(r)=-\pi, r \in\left(r_{0}, 1\right)$, and this solution is the radius of close-toconvexity of $\mathscr{A}_{\alpha}$. For a specific $\alpha, r_{\alpha}$ can be calculated by successive substitutions in $p_{2}(x)$ and $\Delta(r)$.

\section{Univalent subclasses.}

THEOREM 10. If $z f^{\prime}(z)=e^{-i \beta} g(z)[\cos \beta p(z)+i \sin \beta]$ where $g(z)$ is in $\mathscr{F}_{\alpha}$ and $p(z)$ is in $\mathscr{P}$, and if $p(z)$ satisfies either

$$
\left|p(z)-\left(1+t^{2}\right) /\left(1-t^{2}\right)\right| \leqq 2 t /\left(1-t^{2}\right), \quad z \in \Delta, 0<t<1,
$$

or

$$
|\arg [\cos \beta p(z)+i \sin \beta]| \leqq t \pi / 2, \quad z \in \Delta, 0<t<1,
$$

then $f(z)$ is univalent whenever $t$ and $\alpha$ satisfy $t+2 \cos \alpha \leqq 5^{1 / 2}-2$.

Proof. $p_{t}(z)=(1+t z) /(1-t z)$ maps $|z|=1$ onto the circle $\left|w-\left(1+t^{2}\right) /\left(1-t^{2}\right)\right|$ $=2 t /\left(1-t^{2}\right)$, thus if $p(z)$ satisfies $(5.1), p(z)$ is subordinate to $p_{t}(z)$ and there exists a regular function $w(z)$ such that $|w(z)| \leqq|z|$ and $p(z)=p_{t}[w(z)]$. A calculation shows

$$
\frac{z p^{\prime}(z)}{\operatorname{Re}[p(z)]}=\frac{2 t z w^{\prime}(z)}{1-t^{2}|w(z)|^{2}} \cdot \frac{|1-t w(z)|^{2}}{[1-t w(z)]^{2}}
$$

hence, for any real number $\gamma$,

$$
\begin{aligned}
\left|\frac{z p^{\prime}(z)}{p(z)+i \gamma}\right| & \leqq \frac{\left|z p^{\prime}(z)\right|}{\operatorname{Re}[p(z)]} \\
& \leqq \frac{2 t r}{1-r^{2}} \cdot \frac{1-|w(z)|^{2}}{1-t^{2}|w(z)|^{2}}, \quad|z|=r \\
& \leqq \frac{2 t r}{1-r^{2}}
\end{aligned}
$$

where we have used $1-|w(z)|^{2} \leqq 1-t^{2}|w(z)|^{2}$ and $\left|w^{\prime}(z)\right| \leqq\left(1-|w(z)|^{2}\right) /\left(1-|z|^{2}\right)$ [11]. 
If $p(z)$ satisfies (5.2), then there is a $q(z)$ in $\mathscr{P}$ such that for $\mu=\beta / t,|\mu| \leqq \pi / 2$,

$$
\cos \beta p(z)+i \sin \beta=[\cos \mu q(z)+i \sin \mu]^{t} .
$$

$q(z)$ in $\mathscr{P}$ implies $\left|z q^{\prime}(z)\right| /|q(z)+i \gamma| \leqq 2 r /\left(1-r^{2}\right)$ for any real number $\gamma$ [17], hence, differentiating (5.5), we find that in this case $p(z)$ also satisfies (5.4).

Let

$$
h(z)=\frac{f^{\prime \prime}(z)}{f^{\prime}(z)}=\frac{1}{z}\left[\frac{z g^{\prime}(z)}{g(z)}-1\right]+\frac{p^{\prime}(z)}{p(z)+i \tan \beta} .
$$

$\left|(1 / z)\left[z g^{\prime}(z) / g(z)-1\right]\right| \leqq(4 \cos \alpha) /\left(1-r^{2}\right), \quad|z|=r$, follows from the fact that $\operatorname{Re}\left[e^{i \alpha} z g^{\prime}(z) / g(z)\right] \geqq 0$. Consequently, if $g(z) \in \mathscr{F}_{\alpha}$ and $p(z)$ satisfies (5.1) or (5.2), we have $|h(z)| \leqq(4 \cos \alpha+2 t) /\left(1-r^{2}\right)$ which implies $\left|h^{\prime}(z)\right| \leqq 4(4 \cos \alpha+2 t) /\left(1-r^{2}\right)^{2}$ [Problem N, p. 857, Bull. Amer. Math. Soc. 71 (1965)]. Thus if $\{f, z\}$ is the Schwarzian derivative of $f(z)[11]$, then

$$
|\{f, z\}| \leqq\left|h^{\prime}(z)\right|+\frac{1}{2}|h(z)|^{2} \leqq \frac{8(2 \cos \alpha+t)+2(2 \cos \alpha+t)^{2}}{\left(1-r^{2}\right)^{2}}
$$

and, by Nehari's test [12], $f(z)$ is univalent whenever $4(2 \cos \alpha+t)+(2 \cos \alpha+t)^{2}$ $\leqq 1$ which yields the desired result.

\section{BIBLIOGRAPHY}

1. T. Başgöze and F. R. Keogh, The Hardy class of a spiral-like function and its derivative, Proc. Amer. Math. Soc. 26 (1970), 266-269. MR 41 \#8680.

2. G. M. Goluzin, On a variational method in the theory of analytic functions, Leningrad. Gos. Univ. Uč. Zap. 144 Ser. Mat. Nauk 23 (1952), 85-101; English transl., Amer. Math. Soc. Transl. (2) 18 (1961), 1-14. MR 17, 1070; MR 23 \#A1803.

3. P. J. Eenigenburg and F. R. Keogh, On the Hardy class of some univalent functions and their derivatives, Michigan Math. J. 17 (1970), 335-346.

4. J. A. Hummel, $A$ variational method for starlike functions, Proc. Amer. Math. Soc. 9 (1958), 82-87. MR 20 \#1779.

5. - Extremal problems in the class of starlike functions, Proc. Amer. Math. Soc. 11 (1960), 741-749. MR 22 \#11133.

6. W. Kaplan, Close-to-convex schlicht functions, Michigan Math. J. 1 (1952), 169-185. MR 14, 966.

7. J. Krzyż, The radius of close-to-convexity within the family of univalent functions, Bull. Acad. Polon. Sci. Sér. Sci. Math. Astronom. Phys. 10 (1962), 201-204. MR 26 \#6384.

8. R. J. Libera, Univalent $\alpha$-spiral functions, Canad. J. Math. 19 (1967), 449-456. MR 35 \#5599.

9. R. J. Libera and M. R. Ziegler, Regular functions $f(z)$ for which $z f^{\prime}(z)$ is $\alpha$-spiral, Trans. Amer. Math. Soc. (to appear).

10. A. J. Lohwater, G. Piranian and W. Rudin, The derivative of a schlicht function, Math. Scand. 3 (1955), 103-106. MR 17, 249.

11. Z. Nehari, Conformal mapping, McGraw-Hill, New York, 1952. MR 13, 640.

12. - The Schwarzian derivative and schlicht functions, Bull. Amer. Math. Soc. 55 (1949), 545-551. MR 10, 696. 
13. B. Pinchuk, On starlike and convex functions of order $\alpha$, Duke Math. J. 35 (1968), 721-734. MR 37 \#6454.

14. Ch. Pommerenke, On starlike and close-to-convex functions, Proc. London Math. Soc. (3) 13 (1963), 290-304. MR 26 \#2597.

15. M. O. Reade, The coefficients of close-to-convex functions, Duke Math. J. 23 (1956), 459-462. MR 17, 1194.

16. M. S. Robertson, Variational methods for functions with positive real part, Trans. Amer. Math. Soc. 102 (1962), 82-93. MR 24 \#A3288.

17. - Extremal problems for analytic functions with positive real part and applications, Trans. Amer. Math. Soc. 106 (1963), 236-253. MR 26 \#325.

18. - Univalent functions $f(z)$ for which $z f^{\prime}(z)$ is spirallike, Michigan Math. J. 16 (1969), 97-101. MR 39 \#5785.

19. K. Sakaguchi, A variational method for functions with positive real part, J. Math. Soc. Japan 16 (1964), 287-297. MR 31 \#1375.

20. L. Spaček, Přispěvek $k$ teorii funcki prostyčh, Căsopis Pěst. Mat. Fys. 62 (1933), 12-19.

21. M. R. Ziegler, A class of regular functions related to univalent functions, Dissertation, University of Delaware, Newark, Delaware, 1970.

Department of Mathematics, University of Kentucky, Lexington, Kentucky 40506

Current address: Department of Mathematics, Marquette University, Milwaukee, Wisconsin 53233 